\title{
ASSESSMENT OF FACTORS ASSOCIATED WITH DROPOUT FOR PENTAVALENT VACCINE IN TERTIARY CARE HOSPITAL OF KATHMANDU, NEPAL
}

\author{
Shrestha SR, ${ }^{1}$ Shakya $B,{ }^{1}$ Oli $R^{2}$
}

${ }^{1}$ Department of Community Medicine, ${ }^{2}$ Intern, Nepal Medical College Teaching Hospital, Attarkhel, Gokarneshwor-8,

\author{
Kathmandu, Nepal.
}

\begin{abstract}
Immunization is one of the most cost-effective public health measures as it has helped in huge reduction of disease, disability and death from different infectious diseases in children by protecting them from vaccine preventable diseases. The current study aimed to study factors associated with dropout for pentavalent vaccine in tertiary care hospital of Kathmandu. A longitudinal observational study using purposive sampling technique was conducted among 196 infants. The information was obtained using self-constructed structured questionnaire from parents visiting Community Medicine OPD of Nepal Medical College Teaching Hospital. The information regarding socioeconomic characteristics of mother, gender of the infant, place of delivery of child, birth order and reasons for dropout of pentavalent third dose were taken. The dropout of third dose pentavalent vaccine was mainly seen in infant of parents living in rented house and according to gender wise, dropout was high among male infants. The different reasons for dropout of vaccine were busy parents, forgotten date, visit to other immunization centres and sick infants. Among these, the main reason for dropout of vaccine was due to infants getting sick during the time of immunization. The dropout rates between first and third dose of pentavalent vaccine were $26.3 \%$ and $30.4 \%$ respectively for two consecutive months and the overall dropout rate was $28.35 \%$. Also, literacy level of the mother was directly associated with the immunization visit of the infant as, maximum number of infants of illiterate mother were absent for third dose of pentavalent vaccine as opposed to no absentee among master degree holding mothers. Thus, the present study provides valuable information regarding the factors associated with dropout for pentavalent vaccine.
\end{abstract}

\section{KEYWORDS}

Longitudinal observational study, pentavalent vaccine, dropout reasons

\section{CORRESPONDING AUTHOR}

Dr. Supri Raj Shrestha,

Lecturer

Department of Community Medicine,

Nepal Medical College Teaching Hospital,

Attarkhel, Gokarneshwor-8, Kathmandu, Nepal,

Email: supriraj70@gmail.com

Orcid ID: https://orcid.org/0000-0002-8639-5714

DOI: https://doi.org/10.3126/nmcj.v22i3.32624 


\section{INTRODUCTION}

Vaccine is an immuno-biological preparation which produces specific protection against a particular disease. It is a substance which stimulates the production of protective antibody and other immune mechanisms. ${ }^{1}$ Immunization offers an effective health advantage to the children. It is described as the first line of defense against disease. ${ }^{2}$ There is a huge reduction of disease, disability and death from different infectious diseases due to immunization. The individuals who are vaccinated are protected from vaccine preventable diseases and they help in reducing the spread of diseases within the population. ${ }^{3}$ Immunization has eradicated smallpox and it has also helped in reducing morbidity and mortality from vaccine preventable diseases (VPD). ${ }^{4}$ It is estimated that each year about two million deaths occur globally due to VPDs with approximately around 1.5 million occurring in children under five years of age and constituting $15 \%$ of under five deaths. ${ }^{5}$ WHO officially launched a global immunization program in May 1974 which was known as Expanded Program of Immunization for prevention and control of 6 major killer diseases of children, namely tuberculosis, diphtheria, pertussis, tetanus, poliomyelitis and measles, all over the world. ${ }^{6}$

In Nepal, immunization was started in 1979 with the establishment of the Expanded Program on Immunization and this program was gradually expanded and included in all 75 districts by 1989. ${ }^{7}$ This is the most cost-effective public health programme and is also most prioritized program of Nepal. Currently, this immunization program provides vaccination against 11 vaccine preventable diseases. ${ }^{8}$ In world around 34 million children are not completely immunized, with almost $98 \%$ of them living in developing countries. ${ }^{4}$ Immunization during childhood can be considered as an investment in human being as it leads to children's health improvements resulting in their high worker productivity and earnings as adults. Vaccination guards not only against a particular infectious disease, but also helps in providing wide range of health benefits. ${ }^{9}$ In 2018 , globally around $86 \%$ of infants (116.3 million infants) received three doses of diphtheria-pertussis-tetanus (DPT3) vaccine and 129 countries reached around 90\% coverage of DPT3 vaccine. ${ }^{10}$ The drop-out rates of vaccines, for example between DPT1 and DPT3, are defined as the percentage of children that started a course, but couldn't finish the routine immunization for some reason. If the dropout rate of vaccines is low, it indicates good utilization and good service quality. ${ }^{11}$ In Nepal the dropout rate between pentavalent vaccine 1 and pentavalent vaccine 3 is $7.4 \%$ in $2074 / 75 .{ }^{8}$

The different major reasons for dropout of vaccines are postponing it later, child being sick and hence not brought to the centre for immunization, unaware of the importance of immunization, place of immunization being far, no faith in immunization, unaware of the need to return for $2^{\text {nd }}$ and $3^{\text {rd }}$ dose, mother being too busy, fear of side reactions, wrong ideas about immunization and others. ${ }^{4}$

\section{MATERIALS AND METHODS}

This is a longitudinal observational study using purposive sampling technique. The study was conducted among the infants coming for the immunization of first dose of pentavalent vaccine (6 weeks of infant) as per EPI schedule in Community Medicine OPD of Nepal Medical College Teaching Hospital (NMCTH) of two consecutive months, September and October 2019 and was followed on third dose of pentavalent vaccine (14 weeks of infant) during November and December 2019. Ethical approval was taken from Institutional Review Committee (IRC) of NMCTH. The study participants were infants of six weeks who came for the first dose of pentavalent vaccine. Infants who come for immunization except for first and third dose of pentavalent vaccine during the study period were excluded from the study. The parents of the infant of the study period were explained about the purpose of the visit and consent was obtained. Their interview was taken using the self-constructed structured questionnaire and followed on its third dose. During the interview of the parents the information regarding socioeconomic characteristics of mother, gender of the infant, place of delivery of child, birth order and reasons for dropout of pentavalent third dose among those who didn't come according to EPI schedule were asked. The dropout reasons were asked by calling the parents in mobile or telephone. Dropout of pentavalent vaccine were considered among the proportion of children who received the first dose of the pentavalent vaccine at six weeks of age but eventually didn't receive the third dose of the same vaccine at fourteen weeks of age.

Dropout rate of pentavalent vaccine was calculated by (Total number of infants receiving first dose of pentavalent vaccine - Total number of infants receiving third dose of pentavalent vaccine)/ Total number of infants receiving first dose of pentavalent vaccine $* 100 .{ }^{12}$ 
Statistical analysis of the data collected were carried out using SPSS 16. Frequency distribution of dropout reasons of pentavalent vaccine on its third dose were calculated. Chi square test were performed to see the association of immunization status with living status, gender of child, mother's education and mother's occupation. Dropout rate of pentavalent vaccine in NMCTH was also calculated.

\section{RESULTS}

A total of 196 parents were interviewed of infants coming for first dose of pentavalent vaccine during the study period. Out of 196, 140 infants came for the third dose of pentavalent vaccine and 56 infants did not come as per National immunization schedule.

As shown in table 1, out of 196 infants 126 were staying in rent and 70 had their own house. Ninety-two $(73 \%)$ who were staying in rent and 48(68.6\%) who had their own house came for third pentavalent vaccine as per National immunization program.
As shown in table 2, out of 196 infants, 106 male and 90 female came for the first pentavalent vaccine during the study period. Among them,32 (30.2\%) male and 24(26.7\%) female were absent for third dose of pentavalent vaccine as per National immunization program.

As shown in table 3, the different dropout reasons for not coming for third pentavalent vaccine as per National immunization program were infant getting sick (57.2\%), busy parents (21.4\%), visit to other immunization center (14.3\%) and forgotten date (7.1\%).

As shown in table 4, 18 (64.3\%) infants of illiterate mother didn't come for third dose of pentavalent vaccine as per National Immunization Program while 2 (100\%) infants of mother with master degree came. This result shows the association of mother's education with timely visit for immunization of infants.

As shown in table 5, 106 (74.6\%) infants of housewife were brought for third dose of pentavalent vaccine as per National Immunization Program while 8 (57.1\%) infants of mothers who were doing government job were brought .

\section{Table 1: Follow up for third dose of Pentavalent vaccine by type of accommodation}

\begin{tabular}{|lccccc|} 
Type of accommodation & \multicolumn{2}{c}{ Third dose of Pentavalent vaccine } & Chi sq. value & P value \\
& Present N(\%) & Absent N(\%) & Total N(\%) & & \\
Rental & $92(73)$ & $34(27)$ & $126(100)$ & 0.436 & 0.509 \\
House owner & $48(68.6)$ & $22(31.4)$ & $70(100)$ & & \\
Total & $140(71.4)$ & $56(28.6)$ & $196(100)$ & & \\
\hline
\end{tabular}

Table 2: Follow up for third dose of Pentavalent vaccine according to gender of infant

\begin{tabular}{|lccccc|} 
Gender of infant & \multicolumn{2}{c}{ Third dose of Pentavalent vaccine } & Chi sq. value & P value \\
& Present N(\%) & Absent N(\%) & Total (N\%) & & \\
Male & $74(69.8)$ & $32(30.2)$ & $106(100)$ & 0.296 & 0.586 \\
Female & $66(73.3)$ & $24(26.7)$ & $90(100)$ & & \\
Total & $140(71.4)$ & $56(28.6)$ & $196(100)$ & & \\
\hline
\end{tabular}

Table 3: Reasons for dropout of third dose of Pentavalent vaccine

\section{Drop out reason for third \\ Pentavalent vaccine}

$\mathrm{N}(\%)$

Busy parents

12(21.4)

Forgotten date

Visit to other immunization centre

Sick infants

Total

\section{DISCUSSION}

The study conducted by Zewdie A et al between November 2014 and April 2015 in two districts of Hadiya zone of Southern Ethiopia showed that the different reasons for dropout of vaccination were poor counseling of mothers, unsupportive provider-client relationships, poor immunization service arrangements and lack of systems for tracking defaulters. ${ }^{13}$ In the similar study done by Rahman L et al, the reasons for dropping out immunization were lack of awareness to complete the vaccination 
Table 4: Follow up for third dose of Pentavalent vaccine by mother's education

\begin{tabular}{lccccc|} 
Mother's education & \multicolumn{2}{c}{ Third dose of Pentavalent vaccine } & Chi square value & P value* \\
& Present N(\%) & Absent N(\%) & Total N(\%) & & \\
Illiterate & $10(35.7)$ & $18(64.3)$ & $28(100)$ & & \\
Primary & $42(75)$ & $14(25)$ & $56(100)$ & & 0.00 \\
Secondary & $62(86.1)$ & $10(13.9)$ & $72(100)$ & 29.631 & \\
Higher secondary & $22(68.8)$ & $10(31.2)$ & $32(100)$ & & \\
Bachelor & $2(33.3)$ & $4(66.7)$ & $6(100)$ & & \\
Master & $2(100)$ & 0 & $2(100)$ & \\
Total & $140(71.4)$ & $56(28.6)$ & $196(100)$ & \\
\hline
\end{tabular}

*=Likelihood ratio

Table 5: Follow up for third dose of Pentavalent vaccine by mother's occupation

Mother's occupation Third dose of Pentavalent vaccine

Chi square value $P$ value Present N(\%) Absent N(\%) Total N(\%)

Business 26(65) 14(35) 40(100)

Housewife 106(74.6) 36(25.4) 142(100)

2.931

0.231

Government job

8(57.1) 6(42.9) 14(100)

Total 140(71.4) 56(28.6) 196(100)

Table 6: Dropout rates of pentavalent vaccine in Community Medicine OPD of NMCTH

Vaccine

Dropout rate

Pentavalent 1 to Pentavalent $3=$

Total children who came for first pentavalent vaccine in September 2019

followed on November 2019 (91 infants came for pentavalent vaccine first

dose and followed by 67 for third dose)

$26.3 \%$

Total children who came for first pentavalent vaccine in October 2019

followed on December 2019 (105 infants came for pentavalent vaccine first

dose and followed by 73 for third dose)

Overall dropout rate of pentavalent vaccine $=(26.3 \%+30.4 \%) / 2=28.35 \%$

schedule (25\%), illness of child (21.9\%), fear of reaction $(9.4 \%)$ and business of the mother $(9.4 \%){ }^{14}$ In our study, there were total 56 dropouts and the reasons were busy parents 12 (21.4\%), forgotten date $4(7.1 \%)$, visit to other immunization centre $8(14.3 \%)$ and sick child $32(57.2 \%)$.

In the study conducted by Madhavi $\mathrm{N}$ et al, out of t2000 children studied, 1810 were fully immunized in which 974 were male and 836 were female. ${ }^{15}$ In the study conducted by Angadi MM et al, out of 155 children, 54 were fully immunized in which 28 were male and 26 were female. The education level of mothers of those 54 fully immunized children were 23 were uneducated, 5 were primary school completed and 26 were secondary school and above completed. ${ }^{16}$ In our study out of 196 infants, 140 were immunized with third dose of pentavalent vaccine as per EPI schedule in which 74 were male and 66 were female. The education level of mothers of those 140 fully immunized children were 10 were illiterate, 42 were primary school completed, 62 were secondary school completed, 22 were higher secondary school completed, 2 were bachelor level completed and 2 were master level completed.

In the study conducted by Haji A et al, mothers of 1116 children of age $<12$ months, 29 unemployed and 107 employed mothers did not bring their children for their routine immunization of second and third doses of pentavalent vaccines. ${ }^{17}$ In our study out of 196 parents, 36 housewife, 14 business women and 6 government job holder women did not bring 
their children for the third pentavalent vaccine as per EPI schedule.

In the study done by Singh B et al, the dropout rate of DPT1 to DPT3 in Haryana is $3.4 \% .^{18}$ According to Annual Report of Nepal the national dropout rate for Pentavalent 1 vs Pentavalent 3 is 7.4 in FY 2074/75. ${ }^{8}$ In our study dropout rates of pentavalent vaccine are $26.3 \%$ and $30.4 \%$ of two consecutive months and the overall dropout rate is $28.35 \%$. In the similar study done by Singh J et al, dropout rate of DPT1 to DPT3 in Amritsar city is $26.1 \%{ }^{19}$

In conclusion, our study showed that the different reasons for the dropout of third pentavalent vaccine were busy parents, forgotten date of immunization, visit to other immunization centre and infants getting sick on the date of immunization. This study showed that the most common reason for dropout of vaccine was infant getting sick. It may be owed to the fact that the third dose of pentavalent vaccine was given during the winter season. Our study also shows the relation between mother's education to visit for immunization as maximum number of absentees were among infants of illiterate mothers as opposed to no absentees amongst master degree holding mothers.

\section{REFERENCES}

1. Park K. Principles of epidemiology and epidemiologic methods. Park's textbook of preventive and social medicine (24 ed.). M/s Banarsidas Bhanot publishers. 2017; 108.

2. Phukan RK, Barman MP and Mahanta J. Factors associated with immunization coverage of children in Assam, India: over the first year of life. J Trop Pediatr 2009; 55: 249-52.

3. Orensteina WA and Ahmedb R. Simply put: Vaccination saves lives. PNAS 2017; 114: 4031- 3.

4. Vohra R, Vohra A, Bhardwaj P, Srivastava JP and Gupta P. Reasons for failure of immunization: A cross-sectional study among 12-23-month-old children of Lucknow, India. Adv Biomed Res 2013; 2: 1-7.

5. Kaoje AU, Yahaya M, Olayinka RM, Hauwau S, Abubakar J, Ambursa AH. Prevalence of vaccine preventable diseases and utilization of routine immunizations services by parents of under one children in a semi-urban community of Sokoto State, Nigeria. GJMEDPH 2017; 6: 1-10.

6. Suryakantha AH. Epidemiology od infectious disease. Community medicine with recent advances (4 ed). Jaypee the health science publisher. 2017; 290.

7. Vandelaer J, Partridge J, Suvedi BK. Process of neonatal tetanus elimination in Nepal. J Public Health 2009; 31: 561-5.

8. Child Health and Immunization. DoHS, Annual Report 2074/75; 25-48.

9. Fonseca, W., Kirkwood, BR, Victora, CG, Fuchs, SR, Flores, JA and Misago, C. Risk factors for childhood pneumonia among the urban poor in Fortaleza, Brazil: a case-control study. Bull. World Health Organization 1996; 74: 199-208.

10. World Health Organization. Immunization coverage 2019.

11. World Health Organization. Analysis and use of health facility data: guidance for immunization programme managers working document 2018; 16.
12. Legesse E and Dechasa W. An assessment of child immunization coverage and its determinants in Sinana District, Southeast Ethiopia. BMC Pediatrics 2015; 15: 1-14

13. Zewdie A, Letebo M and Mekonnen T. Reasons for defaulting from childhood immunization program: a qualitative study from Hadiya zone, Southern Ethiopia. BMC Public Health 2016; 16: 1240.

14. Rahman L, Biswas H, Hossain T, Khan AM and Khan IA. Study on reasons of dropout of immunization in children in selected slum area of Dhaka city, Bangladesh. South East Asia J Public Health 2012; 2: 64-7.

15. Madhavi N, Manikyamba D. Evaluation of immunization status and factors responsible for drop outs in primary immunization in children between 1-2 years - a hospital based study. Int'l J Pediatr Res 2016; 3: 329-34

16. Angadi MM, Jose AP, Udgiri R, Masali KA and Sorganvi. A study of knowledge, attitude and practices on immunization of children in urban slums of Bijapur city, Karnataka, India. J Clin Diag Resc 2013; 7: 2803-6.

17. Haji A, Lowther S, Ngan'ga Z, Gura Z, Tabu C, Sandhu $\mathrm{H}$ and Arvelo W. Reducing routine vaccination dropout rates: evaluating two interventions in three Kenyan districts, 2014. BMC Public Health 2016; 16:152.

18. Singh B, BMV, Panda M and Khanna P. Study to find out the coverage evaluation and dropout rates of different vaccines in an urban area of Rohtak city in Haryana. Int'l J Basic Applied Med Sci 2013; 3: 223-9.

19. Singh J, Deepti SS, Mahajan S, Lal M, Singh T, Neki NS. Evaluation of vaccination coverage and Dropout rates among children of age 0-5 years in slums of Amritsar city. Int'l J Curr Res Biol Med 2018; 3: 16-22. 